\title{
EXPERIENCE WITH A LOW EMITTANCE OPTICS IN LEP
}

\author{
D. Brandt, W. Herr*, M. Lamont, M. Meddahi, A. Verdier and L. Vos \\ CERN, Geneva, Switzerland
}

\begin{abstract}
Since start-up in 1998, LEP has operated with a low emittance lattice with a phase advance of $102^{\circ}$ in the horizontal and $90^{\circ}$ in the vertical planes. This optics provides a horizontal detuning with amplitude which is small enough to avoid a reduced dynamic aperture in the horizontal plane, a problem experienced in other low emittance lattices. The optics is designed to operate at the highest LEP energies up to and above $100 \mathrm{GeV}$, as well as at $45.6 \mathrm{GeV}$ (still required to provide $Z^{0}$ s for the calibration of the experiments' detectors). The experience gained with this low emittance lattice after one year of operation is presented and its future potential is discussed.
\end{abstract}

\section{PREVIOUS EXPERIENCE WITH LOW EMITTANCE OPTICS}

In order to obtain the highest luminosity with LEP at its highest energies the smallest possible beam emittances are required. Besides providing smaller horizontal emittance, higher horizontal phase advance $\left(\mu_{x}\right)$ in the arc FODO cells has the additional advantage of a smaller momentum compaction thereby increasing the maximum energy attainable with a given RF voltage. A considerable amount of work has been done over the past years to find the best possible lattice and various optics with $90^{\circ} \leq \mu_{x} \leq 135^{\circ}$ have been tested ([1] to [5]). The vertical phase advance per cell used was either $\mu_{y}=60^{\circ}$ or $90^{\circ}$ to make it possible to correct the vertical non-linear chromaticity associated with the low- $\beta$ insertions [6]. The choice of $\mu_{x}$ resulted from an optimisation of the horizontal detuning with amplitude, as the non-linear horizontal chromaticity is much less important than the vertical.

The first lattice developed and tested had $135^{\circ}$ per cell in the horizontal and $60^{\circ}$ in the vertical plane (in short $135^{\circ} / 60^{\circ}$ ). However in preliminary tests no circulating beam could be established; the problems associated with this lattice were studied extensively [7]. It was found impossible to correct both the third order resonance and the derivative of the horizontal tune with respect to the horizontal amplitude. The horizontal detuning for this lattice was $-1.6 \times 10^{5} \mathrm{~m}^{-1}$.

In 1994 , a $108^{\circ} / 60^{\circ}$ lattice was developed. The horizontal detuning was $2.26 \times 10^{4} \mathrm{~m}^{-1}$. This is a factor of seven smaller than the horizontal detuning of the $135^{\circ} / 60^{\circ}$ lattice. After successful tests the $108^{\circ}$ lattice was recommended

\footnotetext{
*Email: Werner.Herr@cern.ch
}

for operation in 1996 [2]. In parallel, a $108^{\circ} / 90^{\circ}$ optics was developed which had a smaller cross detuning term (horizontal detuning with respect to vertical amplitude and vice versa) than the $108^{\circ} / 60^{\circ}$ optics, resulting in a larger horizontal dynamic aperture. Both optics still had a strong horizontal detuning and it was demonstrated that this detuning can bring particles onto non-linear resonances, in particu$\operatorname{lar} 3 Q_{x}$ [7]. As a consequence, operation with $\mu_{x}=108^{\circ}$ optics was hampered by the presence of strong tails, even with a single beam in the machine [8].

The objective was therefore to find an optics with a reduced horizontal detuning. In reducing $\mu_{x}$ from $108^{\circ}$ to $102^{\circ}$, the horizontal detuning decreases by more than a factor of two. The cross detuning of the $102^{\circ} / 90^{\circ}$ lattice is smaller than that of the 1997 operational lattice $\left(90^{\circ} / 60^{\circ}\right)$ by a factor 2 .

Although $\mu_{x}=102^{\circ}=17 \pi / 30$ is not a simple fraction of $\pi$, the excitation term of the systematic 3rd order resonance is smaller than for the $90^{\circ} / 60^{\circ}$ lattice [9].

Measurements with the $102^{\circ} / 90^{\circ}$ were first performed in 1997 with a detuned optics $\left(\beta_{y}^{*}=27 \mathrm{~cm}\right)$ for which chromatic errors were corrected by using only one sextupole family in each plane. After encouraging results, a test under physics conditions was performed with a squeezed optics $\left(\beta_{y}^{*}=5 \mathrm{~cm}\right)$. This necessitated a re-cabling of the vertical sextupole families from three to two families $\left(90^{\circ}\right.$ in the vertical plane instead of $60^{\circ}$ previously used). In the horizontal plane, with a $\beta_{x}^{*}=2 \mathrm{~m}$, one horizontal sextupole family was enough to correct for the non-linear horizontal chromaticity. A successful test was made where the performance with colliding beams was in good agreement with expectation [10].

For 1998 , the possibility of reducing $\beta_{x}^{*}$ from $2.0 \mathrm{~m}$ to $1.25 \mathrm{~m}$ was exploited, increasing the operational flexibility. In this case, the horizontal non-linear chromaticity was corrected by grouping the horizontally focusing sextupoles into several families. The grouping of the families was optimised so that the correction of the horizontal nonlinear chromaticity reduces the horizontal detuning without increasing either the vertical or the cross detuning terms. Beside this, two sextupoles per octant are powered independently to allow a further reduction of the horizontal detuning down to a value 20 times smaller than that of the $108^{\circ}$ optics. The $102^{\circ} / 90^{\circ}$ optics developed for 1998 allows a variation of the horizontal detuning between 0.11 to $1.4 \times 10^{4} \mathrm{~m}^{-1}$. 


\section{EXPERIENCE IN 1998}

The high energy running at LEP in 1998 was performed exclusively with the $102^{\circ} / 90^{\circ}$ optics and at a beam energy of $94.5 \mathrm{GeV}$.

\subsection{Optics - observations}

Horizontal emittance and $\mathbf{J}_{x}$ At $100 \mathrm{GeV}$, operating on central orbit, the $90^{\circ} / 60^{\circ}$ lattice has a horizontal emittance of $56 \mathrm{~nm}$, while the $102^{\circ} / 90^{\circ}$ has an emittance of $44 \mathrm{~nm}$. This means that all other parameters equal, the $102^{\circ} / 90^{\circ}$ has the potential to provide a luminosity 1.3 times larger than that of $90^{\circ} / 60^{\circ}$. It also gives almost $0.6 \mathrm{GeV}$ more centre of mass energy for the same RF voltage.

One way to further reduce the emittance is to increase the horizontal damping partition number $\left(\mathrm{J}_{x}\right)$ through a RF frequency shift. For the $90^{\circ} / 60^{\circ}$, the variation of $J_{x}$ with the momentum shift $\left(\partial J_{x} / \partial \Delta p / p\right)$ was 312 and is 305 for the $102^{\circ} / 90^{\circ}$. In 1998 , the $102^{\circ} / 90^{\circ}$ was operated with a $\mathrm{RF}$ frequency shift of $+120 \mathrm{~Hz}$, i.e a $\mathrm{J}_{x}$ of 1.67 and therefore a horizontal emittance of around $23.6 \mathrm{~nm}$ at $94.5 \mathrm{GeV}$.

Transverse tails Transverse beam tails have been measured with the $102^{\circ}$ optics under physics data taking conditions [11]. The horizontal tails measured at high energies were normally acceptable. However, nonGaussian tails have been observed during so-called background storms [14].

Ramp \& Squeeze The recent years of LEP operation have seen a combined ramp and squeeze in which the vertical squeeze was made during the ramp from 42 to $44 \mathrm{GeV}$. This year, with the wigglers on, some lifetime problems were observed between 50 and $60 \mathrm{GeV}$. A possible cause was an insufficient momentum acceptance of the squeezed $102^{\circ} / 90^{\circ}$ optics. With a $\sigma_{E} / E=0.18 \%$ in the incriminated energy region, the criteria of $8 \sigma_{E} / E$ absolute momentum deviation to guarantee the linear stability is marginal with the squeezed optics but entirely fulfilled with the unsqueezed optics. To avoid this the squeeze was implemented after the wigglers have been switched off at high energy; the lifetime problem was cured. The long range beam-beam effects at the IPs remained acceptably small, despite the increased $\beta_{y}^{*}[12]$.

Minimum $\beta_{x}^{*}$ and $\beta_{y}^{*} \quad$ Background considerations due to the aperture in the horizontally focusing quadrupoles near the experiments limit the minimum $\beta_{x}^{*}$. For $\mathrm{J}_{x}=1$, the limit is $1.21 \mathrm{~m}$ at $100 \mathrm{GeV}$, which is close to the operational value $(1.25 \mathrm{~m})$ [13]. It was shown [14] that the background storms observed during physics fills and cured by small horizontal tune changes, are less severe for larger $\beta_{x}^{*}$, another argument against a further reduction of this value.

Beam-beam effects demand that $\beta_{y}^{*}$ should be larger than $2 \sigma_{l}$ to avoid affecting the luminosity [15]. For $\sigma_{l}=$ $10 \mathrm{~mm}$, the limit on $\beta_{y}^{*}$ is $2 \mathrm{~cm}$, compared to the present value of $4 \mathrm{~cm}$.
A further reduction might be tested to evaluate its operational feasibility. However an attempt to match the physics optics for $\beta_{y}^{*}=3.5 \mathrm{~cm}$ showed that $\beta_{y}$ values were increasing at the collimators near the experiments and that the necessary sextupole strength was severely increased. Beside limiting this $3.5 \mathrm{~cm}$ optics to $95 \mathrm{GeV}$, the large sextupole strength enhances excitation of non-linear resonances and increases the tune dependence with amplitude. Both effects reduce the dynamic aperture [5]. With the $3.5 \mathrm{~cm}$ optics, the non-linear chromaticity correction showed a reduced aperture in momentum $\Delta p / p= \pm 0.013$ (originally \pm 0.015 ) and it was demonstrated that a further reduction of the $\beta_{y}^{*}$ would lead to marginal momentum aperture [16]. In conclusion, $\beta_{x}^{*}$ of $1.25 \mathrm{~m}$ and $\beta_{y}^{*}$ of $4 \mathrm{~cm}$ are very close to the limit for the horizontal and vertical squeeze and will most likely be the standard values for future operation.

\subsection{Experience at $45.6 \mathrm{GeV}$}

After the new $102^{\circ} / 90^{\circ}$ optics was successfully commissioned, the calibration of the LEP detectors on the $\mathrm{Z}_{0}$ peak at $45.6 \mathrm{GeV}$, needed every year, was performed with this lattice at relatively low intensities (to minimise beambeam effects linked to the low emittance). Previous experience with low emittance lattices at $45.6 \mathrm{GeV}\left(108^{\circ}\right.$ optics) showed that performance was limited by the presence of tails. The $102^{\circ}$ optics, with its reduced detuning, did not suffer from the same problems. Physics was performed with trains of $8+8$ bunches and the intensity was limited to $250 \mu \mathrm{A}$ per bunch, in order to keep an acceptable horizontal beam-beam parameter. The luminosity and the vertical beam-beam parameter were exactly as expected. The vertical beam-beam parameter was $0.02-0.025$ and the emittance ratio between 1 and $1.5 \%$. The $102^{\circ}$ optics is the only low emittance LEP lattice which proved to work at low as well as high energies.

\subsection{Experience at $94.5 \mathrm{GeV}$}

At high energy, three different physics periods can be differentiated, depending on the value of the beta functions at the interaction points. During the first period (up to fill 5140, Fig. 1$), \beta_{x}^{*}=1.5 \mathrm{~m}$ and $\beta_{y}^{*}=5 \mathrm{~cm}$ were used and both $\xi_{y}$ and the peak luminosity were increasing as the total current was increased. For the second period (fills 5140-5262), $\beta_{x}^{*}$ was decreased to $1.25 \mathrm{~m}$ while $\beta_{y}^{*}$ remained unchanged. Both $\xi_{y}$ and the peak luminosity were still increasing without any further increase of the total current. Finally, from fill 5263 onwards, $\beta_{y}^{*}$ was reduced to $4 \mathrm{~cm}$ while $\beta_{x}^{*}$ remained unchanged. The result was a decrease of $\xi_{y}$ and an increase of the luminosity compared to the second period, with a similar total current.

In order to quantify these changes and to check whether the effects of the horizontal and vertical squeeze were as expected, we compare one representative fill in each of the three physics periods (see table 1).

For the horizontal squeeze, for the same bunch intensity, squeezing the horizontal beta value from $1.5 \mathrm{~m}$ to 


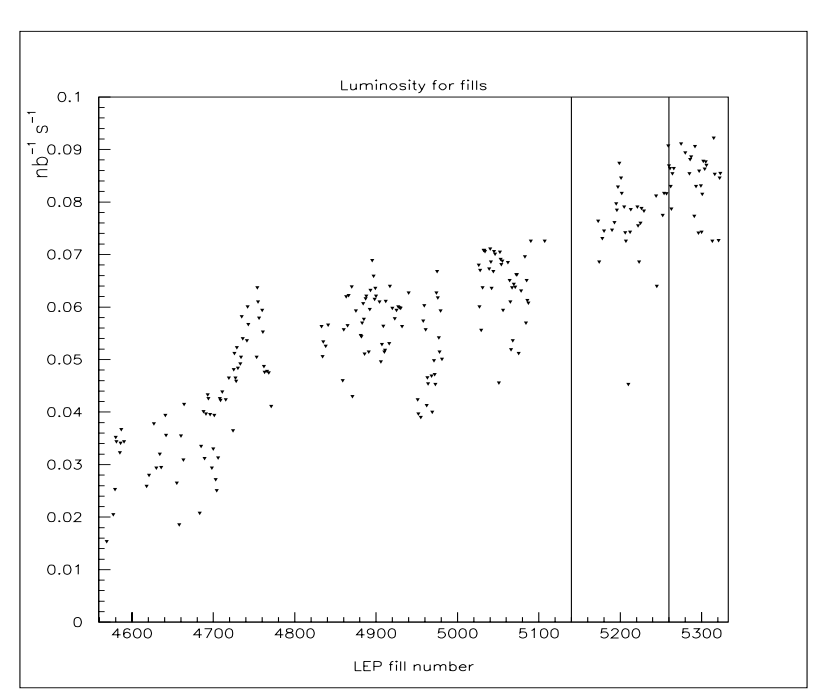

Figure 1: Luminosity as a function of fill number

\begin{tabular}{||l|c|c|c|c|c|c||}
\hline \hline $\begin{array}{l}\beta_{x}^{*} \\
\mathrm{~m}\end{array}$ & $\begin{array}{c}\beta_{y}^{*} \\
\mathrm{~cm}\end{array}$ & $\xi_{y}$ & $\xi_{x}$ & $\begin{array}{c}\text { Lum. } \\
\times 10^{31}\end{array}$ & $\begin{array}{c}\mathrm{I} / \text { bunch } \\
\mu \mathrm{A}\end{array}$ & $\begin{array}{c}\Delta \mathrm{f}_{r f} \\
\mathrm{~Hz}\end{array}$ \\
\hline 1.50 & 5 & 0.062 & 0.042 & 7.3 & 750 & 0 \\
1.25 & 5 & 0.076 & 0.042 & 9.0 & 750 & 120 \\
1.25 & 4 & 0.061 & 0.042 & 9.5 & 750 & 120 \\
\hline \hline
\end{tabular}

Table 1: Three fills during the different running periods

$1.25 \mathrm{~m}$ and increasing the RF frequency shift from $100 \mathrm{~Hz}$ to $120 \mathrm{~Hz}$, is expected to give $20 \%$ increase in both the $\xi_{y}$ and peak luminosity. The expectations were effectively met. For the same bunch intensity, and a vertical squeeze from $5 \mathrm{~cm}$ to $4 \mathrm{~cm}$, one expects $11 \%$ decrease in $\xi_{y}$ and $11 \%$ increase in luminosity. The expectations were met in $\xi_{y}$ and were very close in luminosity [13].

High beam-beam parameters were regularly achieved and did not show any sign of saturation with increasing current. This is extensively treated in [17].

\section{FUTURE PERFORMANCE}

All magnets and power converters allow the $102^{\circ} / 90^{\circ}$ optics to operate up to $103 \mathrm{GeV}$. In 1999 , the performance limitations will depend on the maximum possible intensity and on the highest reliable accelerating gradient [18]. For 2000 , the cryogenics cooling power will also be a limitation for energies above $100 \mathrm{GeV}$. Performance estimates for 1999 foresee an integrated luminosity of $1.5 \mathrm{pb}^{-1} /$ day for $6 \mathrm{~mA}$ total current. With $8 \mathrm{~mA}, 2 \mathrm{pb}^{-1} /$ day are expected. However it should be emphasised that for high intensity and high energy the global efficiency is likely to be reduced. A word of caution has to be added regarding the available aperture: it remains to be demonstrated that a horizontal emittance of $47.3 \mathrm{~nm}$ at $103 \mathrm{GeV}$ can be accommodated in the machine. Also, the available momentum aperture, which is limited by the non-linear vertical chromaticity correction, was shown to be marginal above
$100 \mathrm{GeV}$ [16]. Optimisation of the phases of the experimental interaction regions and/or vertical phase advance in the arcs can be made in order to improve the non-linear chromaticity correction.

\section{CONCLUSIONS}

In 1997, a low emittance optics with a phase advance of $102^{\circ}$ per cell in the horizontal and $90^{\circ}$ in the vertical planes was developed and commissioned. This optics has significantly smaller detuning with amplitude than previously tested low emittance lattices with larger horizontal phase advance. As a consequence it has less operational problems. In 1998, it was used for the whole year in operation and the performance was excellent. The $102^{\circ} / 90^{\circ}$ optics gives a slightly higher energy for a given total RF voltage and has a potentially higher performance at the highest energy achievable at LEP. It is the first low emittance lattice which proved to work for both low and high energies.

\section{REFERENCES}

[1] D. Brandt et al., Proc. 3rd Eur. Part. Acc. Conf., Berlin 1992.

[2] Y. Alexahin et al, Proc. Part. Acc. Conf., Dallas 1995.

[3] Y. Alexahin et al, Proc. 5th Eur. Part. Acc. Conf., Sitges 1996.

[4] D. Brandt et al., Proc. Part. Acc. Conf., Vancouver 1997.

[5] D. Brandt et al; Proc. 6th Eur. Part. Acc. Conf., Stockholm 1998.

[6] A. Verdier, Proc. Part. Acc. Conf., Dallas 1995.

[7] Y. Alexahin, CERN SL/95-110 (AP), 1995.

[8] H. Burkhardt, Proc. 8th LEP performance workshop, CERN SL/98-06.

[9] A. Verdier, Proc. 7th LEP performance workshop, CERNSL/97-06.

[10] M. Meddahi; Proc. 8th LEP performance workshop, CERN SL/98-06.

[11] K. Affholderbach et al; SL-Note-98-073 (MD).

[12] D. Brandt and M. Lamont, SL-Note-98-070(MD).

[13] M. Meddahi; Proc. 9th LEP performance workshop. CERNSL/99-07.

[14] G. von Holtey; Proc. 9th LEP performance workshop. CERN-SL/99-07.

[15] S. Myers; Inst. and Meth. 211 (1983) 263-282, North Holland Publishing Company.

[16] A. Verdier; Proc. 9th LEP performance workshop. CERNSL/99-07.

[17] D. Brandt et al.; Is LEP beam-beam limited at its highest energy?; These Proceedings.

[18] D. Brandt, S. Myers, CERN SL/98-074 (DI). 